\title{
Interaction-energy functional of the Hubbard model: Local formulation and application to low-dimensional lattices
}

\author{
Matthieu Saubanère, ${ }^{1}$ Marie Bernadette Lepetit, ${ }^{2}$ and G. M. Pastor ${ }^{3}$ \\ ${ }^{1}$ Institut Charles Gerhardt, Centre National de la Recherche Scientifique, Université de Montpellier, \\ Place Eugène Bataillon, 34095 Montpellier, France \\ ${ }^{2}$ Institut Néel, UPR CNRS 2940, 25 rue des Martyrs, 38042 Grenoble cedex 9, France \\ ${ }^{3}$ Institut für Theoretische Physik, Universität Kassel, Heinrich Plett Straße 40, 34132 Kassel, Germany \\ (Received 21 January 2016; revised manuscript received 13 June 2016; published 5 July 2016)
}

\begin{abstract}
The interaction energy $W[\gamma]$ of the Hubbard model is regarded as a functional of the single-particle density matrix $\gamma$ in the framework of lattice density-functional theory. The local character of the Hubbard interaction is exploited to express $W$ as a sum of local contributions $\omega_{i}[\gamma]$, for which a simple semilocal scaling approximation is proposed. The method is applied to the ionic Hubbard model on one- and two-dimensional lattices with homogeneous and inhomogeneous Coulomb repulsions. Results are given for the kinetic and Coulomb energies, interatomic charge transfers, local magnetic moments, and charge gaps. Goals and limitations of the functional are discussed by comparison with exact results.
\end{abstract}

DOI: 10.1103/PhysRevB.94.045102

\section{INTRODUCTION}

Density functional theory (DFT) has revolutionized our perspective to the electronic properties of matter [1]. Replacing the wave function by the electronic density $\rho(\vec{r})$ as the basic variable of the many-body problem has been a major practical and conceptual breakthrough. Local and semilocal approaches to the interaction-energy functional have been most appealing from the start, particularly due to their simplicity and flexibility. Indeed, the local density approximation (LDA) based on the homogeneous electron gas has been the key to the early success of DFT [2,3]. In the meantime, the theory has found an incredibly large number of successful applications in the most broad variety of fields. Moreover, it has been the subject of significant methodological clarifications and developments [4], including generalized-gradient approximations [5], hybrid functionals [6-10], as well as density-matrix approaches [11-19]. Nevertheless, a number of open problems prevail, in particular in the context of strongly correlated systems [20] (e.g., Mott insulators, narrow-band systems, heavy-fermions materials, and high- $T_{c}$ superconductors) where the conventional exchange and correlation functionals fail systematically. Finding an accurate DFT description of these materials is therefore challenging [21-25].

The present understanding of strong electron correlations in condensed-matter physics relies for the most part on lattice models, which aim to capture the subtle low-energy manybody physics [26]. Among the most famous and basic examples in this field, one should mention the Anderson impurity model [27] and the Hubbard model [28], which pointed the way to countless extensions of growing richness and complexity [26]. The central feature of these Hamiltonians is their ability to describe the interplay between electron delocalization, due to various hybridizations, and the concomitant fluctuations of the local charges and Coulomb-repulsion energy. In this way, subtle many-body effects, such as correlation-induced localization, local spin and valence fluctuations, and the separation of spin and charge degrees of freedom can be understood. Picturing this kind of phenomena on the basis of the electronic density $\rho(\vec{r})$ seems difficult a priori, despite the incontestable general validity of the Hohenberg-Kohn (HK) theorem.

Although many-body lattice models are strongly simplified in comparison with the first principles Hamiltonian, elucidating their properties is far from trivial. Only very few exact analytical results are available [29-33] and straightforward numerical solutions are rapidly blocked by the exponential increase of the Hilbert-space dimension with system size. Consequently, a large variety of approximations has been developed [26]. Since DFT is a universal theory, it should be possible to apply it to lattice models. In fact, several investigations have been done by adapting the concepts of DFT to lattice Hamiltonians. Some authors have studied the band gap problem in semiconductors [34], the role of offdiagonal elements of the density matrix in strongly correlated systems [35]. Others have proposed and applied density-matrix energy functionals to the Hubbard and Anderson models [36-39], or have derived local approximations based on the exact solutions for one-dimensional (1D) systems [40]. More recently, progress has been made on time-dependent approaches [41] and on the description of attractive local interactions $[42,43]$.

The staring point of DFT in the continuum has been to realize that the single-particle external potential $v(\vec{r})$ acting on the electrons defines the problem under study, while the form and parameters involved in the kinetic and interaction energies of the electronic system are universal. In lattice models, the single-particle hopping matrix $t_{i j}$ defines the system under consideration, since it defines the lattice structure, its dimensionality and connectivity matrix. In the continuum, $v(\vec{r})$ couples to $\rho(\vec{r})$, while in the lattice, $t_{i j}$ couples to the single-particle density matrix $\gamma_{i j}$. Consequently, $\gamma$ naturally emerges as the fundamental variable for lattice density-functional theory (LDFT). In fact, a lattice version of the HK theorem showing the one-to-one mapping between $t_{i j}$ and the ground-state $\gamma_{i j}$ has been demonstrated [36]. A similar situation is also found in the continuum when one considers nonlocal pseudopotentials [11]. More recently, functionals of the single-particle density matrix $\rho\left(\vec{r}, \vec{r}^{\prime}\right)$ have been proposed in order to study problems with local potentials $v(\vec{r})$. Even 
though this is not required by the HK theorem, an improved performance is expected [12-19].

As in conventional DFT, the central challenge in LDFT is to find accurate simple approximations to the correlation-energy functional $W[\gamma]$. In early studies, $W[\gamma]$ has been described by using the Hubbard dimer as a reference system and by taking advantage of the scaling properties of $W$ under the assumption of an homogeneous charge distribution $\gamma_{i i}$ [44-47]. Although the scaling ideas have been extended to bipartite lattices [48,49], the latter assumption appears as a limitation, since it precludes applications to problems lacking translational symmetry (e.g., finite clusters, surfaces, disordered systems, etc.). It is the main purpose of the present paper to introduce a local formulation of the interaction energy functional $W[\gamma]$, which can be applied to general models having on-site interactions by keeping the advantages and simplicity of the scaling approach. In order to quantify the performance of the method, we apply it to homogeneous and ionic Hubbard models testing its accuracy by comparison with various alternative approaches.

The remainder of the paper is organized as follows. In Sec. II the basic principles of the density functional theory of lattice models are recalled. The interaction energy of Hubbard-like models is expressed as a sum of local contributions, which are functionals of the single-particle density matrix $\gamma$. The self-consistent equations which implement the variational principle and give access to the ground-state properties are reproduced. In Sec. II C we propose a simple iterative algorithm in order to solve the LDFT equations. This numerical procedure, to be used in our calculations, can also be applied straightforwardly to density-matrix functional theories in the continuum [11]. Section III introduces a simple semilocal scaling approximation to $W[\gamma]$ of the ionic Hubbard model. This functional is then used to determine the ground-state kinetic and Coulomb energies, interatomic charge transfers, local magnetic moments, and charge gaps of one- and twodimensional systems. In Sec. IV we focus on homogeneous systems and compare our results with those obtained using the density-matrix renormalization group (DMRG), quantum Monte Carlo (QMC), and slave-boson mean-field (SBMF) approaches [50-52]. The case of inhomogeneous Coulomb repulsions is discussed in Sec. V. Finally, in Sec. VI we investigate the crossover from band to Mott insulators in the ionic Hubbard model. Goals and limitations of the functional are discussed by comparison with DMRG and QMC calculations.

\section{DENSITY-MATRIX FUNCTIONAL THEORY ON A LATTICE}

In the following we recall the basics of LDFT in the context of the inhomogeneous Hubbard model, giving emphasis to the local formulation of the interaction-energy functional and to the variational equations from which the ground-state behavior is obtained. A general discussion of the density-functional theory of lattice models and minimal basis Hamiltonians may be found in Ref. [53].

The ionic Hubbard model is given by [28]

$$
\hat{H}=\sum_{i, \sigma} \varepsilon_{i} \hat{n}_{i \sigma}+\sum_{i, j, \sigma} t_{i j} \hat{c}_{i \sigma}^{\dagger} \hat{c}_{j \sigma}+\sum_{i} U_{i} \hat{n}_{i \downarrow} \hat{n}_{i \uparrow},
$$

where $\varepsilon_{i}$ denotes the energy level at site $i, t_{i j}$ the nearest neighbor (NN) hopping integrals, and $U_{i}$ the local Coulomb repulsions. As usual, the operator $\hat{c}_{i \sigma}^{\dagger}\left(\hat{c}_{i \sigma}\right)$ creates (annihilates) a spin- $\sigma$ electron at site $i$. Thus, $\hat{n}_{i \sigma}=c_{i \sigma}^{\dagger} c_{i \sigma}$ is the corresponding number operator. The hopping integrals $t_{i j}$ define the dimensionality and structure of the lattice, as well as the range of the single-particle hybridizations. The energy levels $\varepsilon_{i}$ describe either the distribution of different elements in the lattice or the effects of inhomogeneous external fields.

The single-particle model parameters $\varepsilon_{i}$ and $t_{i j}$ specify the system under study and therefore play the role given in conventional DFT to the external potential $v_{\text {ext }}(\vec{r})$. They enter the Hamiltonian $\hat{H}$ linearly through a matrix product with the operators $c_{i \sigma}^{\dagger} c_{j \sigma}$. Therefore, the single-particle density matrix

$$
\gamma_{i j \sigma}=\left\langle\Psi\left|\hat{c}_{i \sigma}^{\dagger} \hat{c}_{j \sigma}\right| \Psi\right\rangle
$$

between the lattice sites $i$ and $j$ may be used to replace the wave function $|\Psi\rangle$ as the basic variable of the many-body problem. Indeed, a lattice version of the Hohenberg-Kohn theorem has been established, which explicitly demonstrates that the mapping between $|\Psi\rangle$ and the single-particle density matrix $\gamma$ is injective and therefore invertible [36]. Consequently, one may regard the ground-state properties as functionals of $\gamma_{i j \sigma}$.

The variational principle implies that the energy $E_{\mathrm{gs}}$ and single-particle density-matrix $\gamma_{\mathrm{gs}}$ of the ground state can be obtained by minimizing the energy functional

$$
E[\gamma]=K[\gamma]+W[\gamma]
$$

with respect to $\gamma=\sum_{\sigma} \gamma_{\sigma}$. Knowing the kinetic energy

$$
K[\gamma]=\sum_{i, \sigma} \varepsilon_{i} \gamma_{i i \sigma}+\sum_{i j, \sigma} t_{i j} \gamma_{i j \sigma}
$$

explicitly, the challenge in LDFT consists in obtaining accurate approximations to the interaction energy $W[\gamma]$. This can be formally expressed by using Levy-Lieb's constrained search as

$$
W[\gamma]=\min _{\Psi \rightarrow \gamma}\left[\sum_{i} U_{i}\left\langle\Psi\left|\hat{n}_{i \uparrow} \hat{n}_{i \downarrow}\right| \Psi\right\rangle\right],
$$

where the minimization runs over all many-body states $|\Psi\rangle$ yielding the density matrix $\gamma$ according to Eq. (2). $W[\gamma]$ thus represents the minimum value of the interaction energy, compatible with a given charge distribution and electron delocalization throughout the lattice.

\section{A. Local expansion of the interaction energy of the Hubbard model}

Denoting by $|\Psi[\gamma]\rangle$ the many-body state which actually minimizes the Coulomb repulsion $W$ in Eq. (5) for the given $\gamma$, we may write

$$
W[\gamma]=\sum_{i} U_{i}\left\langle\Psi[\gamma]\left|\hat{n}_{i \uparrow} \hat{n}_{i \downarrow}\right| \Psi[\gamma]\right\rangle .
$$

Notice that $|\Psi[\gamma]\rangle$ is a universal functional of $\gamma$, which depends on all $U_{i}$ and on band filling but not on the structure or dimensionality of the lattice.

In order to split $W[\gamma]$ as a sum of local contributions, it is useful to regard it as a function of the local Coulomb 
repulsions $\vec{U}=\left(U_{1}, U_{2}, \ldots U_{N}\right)$ at the different lattice sites for fixed $\gamma$. From this perspective, $W$ is a positive homogeneous function of degree one, i.e., $W[\gamma, \alpha \vec{U}]=\alpha W[\gamma, \vec{U}]$ for all $\alpha>0$. Applying Euler's theorem we have

$$
W[\gamma]=\sum_{i} U_{i} \frac{\partial W}{\partial U_{i}} .
$$

Since $W$ is stationary with respect to variations of $|\Psi[\gamma]\rangle$ for the given $\gamma$ and $\vec{U}$, we conclude that

$$
\frac{\partial W}{\partial U_{i}}=\left\langle\Psi[\gamma]\left|\hat{n}_{i \uparrow} \hat{n}_{i \downarrow}\right| \Psi[\gamma]\right\rangle=\omega_{i}[\gamma]
$$

is given by the local average number of double occupations $\omega_{i}[\gamma]$ at site $i$ in the optimal state $|\Psi[\gamma]\rangle$. Consequently, the interaction energy functional

$$
W[\gamma]=\sum_{i} U_{i} \omega_{i}[\gamma]
$$

can be expressed as the sum of local contributions. A further consequence of Euler's theorem is that $\omega_{i}$ is invariant upon a uniform scaling of all Coulomb integrals, i.e., $\omega_{i}[\gamma, \alpha \vec{U}]=$ $\omega_{i}[\gamma, \vec{U}]$ for $\alpha>0$. The latter follows more directly from Eqs. (5) and (8) by noting that $|\Psi[\gamma]\rangle$ is not affected by scaling $\vec{U}$. These are fundamental properties to be fulfilled by any approximation. Notice, however, that $\omega_{i}$ depends in general on all $\gamma_{i j \sigma}$, so that the functional remains nonlocal, as physically expected.

\section{B. Variational equations of lattice density-functional theory}

The density matrix $\gamma$ is characterized by its eigenvectors, or natural spin orbitals $|k \sigma\rangle$, and by its eigenvalues, or occupation numbers $\eta_{k \sigma}$. The Hermiticity of $\gamma$ implies that the kets $|k \sigma\rangle$ form an orthonormal single-particle basis, while the representability of $\gamma$ requires $0 \leqslant \eta_{k \sigma} \leqslant 1$ [45]. Denoting by $u_{i k \sigma}=\langle i \mid k \sigma\rangle$ the component of $|k \sigma\rangle$ at site $i$ we have

$$
\gamma_{i j \sigma}=\sum_{k=1}^{N} u_{i k \sigma} \eta_{k \sigma} u_{j k \sigma}^{*} .
$$

The extrema of $E[\gamma]$, subject to the constraints on normalization and number of particles, are the stationary points of the Lagrange function

$$
\begin{aligned}
\mathcal{L}[\gamma]= & E[\gamma]-\mu \sum_{\sigma}\left(\sum_{k} \eta_{k \sigma}-N_{\sigma}\right) \\
& -\sum_{k, \sigma} \varepsilon_{k \sigma}\left(\sum_{i}\left|u_{i k \sigma}\right|^{2}-1\right),
\end{aligned}
$$

where $\mu$ and $\varepsilon_{k \sigma}$ are Lagrange multipliers, and $N_{\sigma}$ is the number of electrons with spin $\sigma\left(N=N_{\uparrow}+N_{\downarrow}\right)$ [45]. Variation with respect to the natural orbitals yields

$$
\sum_{i}\left(\frac{\partial E[\gamma]}{\partial \gamma_{i j \sigma}} \eta_{k \sigma}-\varepsilon_{k \sigma} \delta_{i j}\right) u_{i k \sigma}=0
$$

for all $j, k$, and $\sigma$, where we have used that

$$
\frac{\partial E[\gamma]}{\partial u_{j k \sigma}^{*}}=\sum_{i} \frac{\partial E[\gamma]}{\partial \gamma_{i j \sigma}} \frac{\partial \gamma_{i j \sigma}}{\partial u_{j k \sigma}^{*}}=\sum_{i} \frac{\partial E[\gamma]}{\partial \gamma_{i j \sigma}} u_{i k \sigma} \eta_{k \sigma} .
$$

This defines the hermitic eigenvalue problem

$$
\sum_{i} \frac{\partial E[\gamma]}{\partial \gamma_{i j \sigma}} u_{i k \sigma}=\lambda_{k \sigma} u_{j k \sigma}
$$

with $\lambda_{k \sigma}=\varepsilon_{k \sigma} / \eta_{k \sigma}$ for $\eta_{k \sigma} \neq 0$ [45]. Vanishing $\eta_{k \sigma}$ are irrelevant here, since in this case $\gamma$ is independent of $u_{j k \sigma}$.

The minimization with respect to the occupation numbers gives

$$
\frac{\partial \mathcal{L}}{\partial \eta_{k \sigma}}=\sum_{i, j} \frac{\partial E[\gamma]}{\partial \gamma_{i j \sigma}} u_{i k \sigma} u_{j k \sigma}^{*}-\mu=\lambda_{k \sigma}-\mu=0,
$$

where the second equality follows from Eq. (13). At the minimum we must thus have $\lambda_{k \sigma}<\mu$ for $\eta_{k \sigma}=1, \lambda_{k \sigma}>\mu$ for $\eta_{k \sigma}=0$, and

$$
\lambda_{k \sigma}=\frac{\partial E}{\partial \eta_{k \sigma}}=\mu
$$

for all natural orbitals having nontrivial fractional occupations $0<\eta_{k \sigma}<1$ [45]. Self-consistency is implied by the dependence of $\partial W / \partial \gamma_{i j \sigma}$ and $\lambda_{k \sigma}$ on $\eta_{k \sigma}$ and $u_{i k \sigma}$. Analogous relations have been derived in the context of density-matrix functional theory in the continuum [11]. Notice, however, the contrast with the usually assumed noninteracting $v$ representability, which implies integer occupations [2] (see also Refs. [4,54]). The importance of fractional occupations to the description of electron correlations within density-matrix functional theory has already been stressed by Gilbert [11].

As an example in the context of the Hubbard model, Fig. 1 shows the ground-state occupation numbers $\eta_{k \sigma}$ in a periodic $1 \mathrm{D}$ ring as a function of the interaction strength $U / t$. These results were obtained by performing exact Lanczos diagonalizations for $N_{a}=14$ sites and half-band filling $n=$ $N / N_{a}=1$ [55]. Each curve corresponds to a different value of $k=(2 \pi / a)\left(v / N_{a}\right)$ with integer $v(0 \leqslant|v| \leqslant 7)$. For fixed $U / t, \eta_{k \sigma}$ decreases with increasing $|k|$, since small $|k|$ values are favored by the kinetic energy $(t<0)$. As $U / t$ increases,

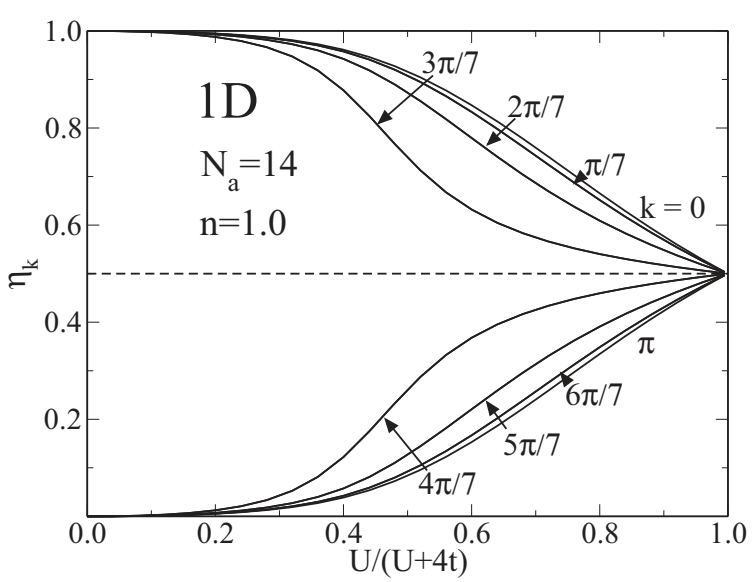

FIG. 1. Exact occupation numbers $\eta_{k \uparrow}=\eta_{k \downarrow}$ of the natural orbitals $|k \sigma\rangle$ in the ground state of a periodic Hubbard ring, having $N_{a}=14$ sites, homogeneous energy levels $\varepsilon_{i}=0$, and half-band filling $n=1$, as a function of the interaction strength $U / t$. The numbers indicate the value of $|k|$ corresponding to each curve $\left(\eta_{k \sigma}=\eta_{-k \sigma}\right)$. 
large $|k|$ antibonding orbitals are occupied at the expense of small $|k|$ bonding orbitals in order to reduce charge fluctuations and the average Coulomb repulsion energy. For $n=1$, all occupations are equal in the strongly-correlated localized limit $(U / t \rightarrow+\infty)$. Notice that all the occupations are fractional, except for $U=0$. Thus, Eq. (15) is the relevant case for realistic applications of LDFT.

\section{Optimizing correlated fractional occupations with an auxiliary noninteracting finite-temperature system}

Before discussing the scaling approximation to $\omega_{i}[\gamma]$ of the ionic Hubbard model, we describe in this section an iterative algorithm in order to solve the LDFT equations, which extends some ideas of the Kohn and Sham approach [2] to the case of fractional occupations. As we shall see, the method could also be applied to reduced-density-matrix functional theories in the continuum. Our goal is to find an auxiliary noninteracting $N$-electron system, whose equilibrium naturalorbital occupations $\eta_{k \sigma}^{a}$ are the same as those of the exact interacting ground state. Since the latter are always fractional, the auxiliary system must be regarded at some fictitious finite temperature $T_{a}$, even though the exact solution concerns the ground state. Given a basis of natural orbitals $|k \sigma\rangle$, the noninteracting system is characterized by the energy levels $\varepsilon_{k \sigma}^{a}$. At equilibrium its occupations minimize the free energy

$F_{a}=\sum_{k \sigma}\left\{\eta_{k \sigma} \varepsilon_{k \sigma}^{a}+T_{a}\left[\eta_{k \sigma} \ln \left(\eta_{k \sigma}\right)+\left(1-\eta_{k \sigma}\right) \ln \left(1-\eta_{k \sigma}\right)\right]\right\}$

for fixed $\varepsilon_{k \sigma}^{a}$, subject to the constraint on the total number of particles. The minimum is of course given by the Fermi distribution

$$
\eta_{k \sigma}^{a}=\frac{1}{e^{\left(\varepsilon_{k \sigma}^{a}-\mu\right) / T_{a}}+1} .
$$

In order that the auxiliary system leads us to the exact groundstate occupations, we request that the gradients of $F_{a}$ and $E$ coincide: $\partial F_{a} / \partial \eta_{k \sigma}=\partial E / \partial \eta_{k \sigma}$. This provides us with the explicit form

$$
\varepsilon_{k \sigma}^{a}=\frac{\partial E}{\partial \eta_{k \sigma}}+T_{a} \ln \left(\frac{1-\eta_{k \sigma}}{\eta_{k \sigma}}\right)
$$

of the auxiliary energy levels as a function of the occupations $\eta_{k \sigma}$. It is easy to verify, by replacing Eq. (18) in (17), that the self-consistency constraint $\eta_{k \sigma}^{a}=\eta_{k \sigma}$ for all $k \sigma\left(T_{a} \neq 0\right)$ is equivalent to the extremal condition $\partial E / \partial \eta_{k \sigma}=\mu$ for all $k \sigma$, where the chemical potential $\mu$ corresponds to the total number of particles $N$ (i.e., $\sum_{k \sigma} \eta_{k \sigma}=N$ ). In this way, the optimal occupations for fixed natural orbitals $|k \sigma\rangle$ are obtained, as stated in Eq. (15). This shows how the exact fractional occupations of the correlated problem can be obtained as the finite-temperature equilibrium values of an effective noninteracting system.

The previous reformulation suggests the following doubleloop iterative scheme for solving the LDFT equations: (i) Find a reasonable starting guess for $u_{i k \sigma}$ and $\eta_{k \sigma}$, for example, from the ground state of the noninteracting Hamiltonian $\left(U_{i}=0\right)$ which has integer occupations but respects the lattice symmetry. (ii) Optimize the orbital occupations $\eta_{k \sigma}$ by keeping the current $u_{i k \sigma}$ fixed. To this aim, determine the auxiliary single-particle levels $\varepsilon_{k \sigma}^{a}$ from Eq. (18), obtain $\eta_{k \sigma}^{a}$ from (17), and update with them $\eta_{k \sigma}$. Iterate until convergence is achieved (i.e., $\eta_{k \sigma}^{a}=\eta_{k \sigma}$ ). (iii) Obtain $\gamma_{i j \sigma}$ and $\partial E[\gamma] / \partial \gamma_{i j \sigma}$ for the current $u_{i k \sigma}$ and the self-consistent $\eta_{k \sigma}$ from (ii). Find new natural orbital $u_{i k \sigma}^{\prime}$ by solving the eigenvalue problem (13). (iv) Compare $u_{i k \sigma}^{\prime}$ with the previous $u_{i k \sigma}$ and exit if convergence has been achieved (i.e., $u_{i k \sigma}^{\prime}=u_{i k \sigma}$ ). Otherwise return to (ii) after having updated $u_{i k \sigma}$ with $u_{i k \sigma}^{\prime}$.

This simple iterative algorithm proves to be quite stable and effective, at least for the applications and functionals considered in this paper. Nevertheless, extreme values of $T_{a}$ are to be avoided, since they result either in steplike or in very flat Fermi functions. Setting simply $T_{a} \simeq t$ is already a good choice in most cases. Moreover, $T_{a}$ may be tuned to some extent, depending on the model, band filling $n$, and interaction strength $U / t$, in order to improve the numerical stability and reduce the number of iterations [56].

\section{SEMILOCAL SCALING APPROXIMATION TO $W[\gamma]$}

The purpose of this section is to derive a semilocal approximation to the interaction-energy functional $W$, which is based on the local expansion (9) and can be applied to general Hubbard-like models. For simplicity we restrict ourselves to non-spin-polarized systems by assuming $\gamma_{i j \uparrow}=\gamma_{i j \downarrow}=\gamma_{i j} / 2$ throughout. Given a lattice structure, band filling $n$, and charge distribution $\gamma_{i i}$, the ground state $\gamma_{i j}^{\mathrm{gs}}$ is bounded by the system-specific $v$ representability domain $\gamma_{i j}^{\infty} \leqslant \gamma_{i j} \leqslant \gamma_{i j}^{0}$ for $i \neq j$. The lower bounds $\gamma_{i j}^{\infty}$ represent the maximum hopping rates or bond orders, which are compatible with the minimum average number of double occupations, and the upper bounds $\gamma_{i j}^{0}$ represent the largest hopping rates without any restrictions. The former are reached in the strongly correlated limit, where the double occupations $\omega_{i}=\omega_{i}^{\infty}$ are minimal, while the latter correspond to the noncorrelated limit, where the double occupations $\omega_{i}=\omega_{i}^{0}$ are maximal. In order to derive transferable functional relations between $\omega_{i}$ and $\gamma_{i j}$, it is useful to scale these properties within the domain of $v$ representability of $\gamma$. One therefore considers dimensionless site-dependent quantities, which locally measure the degree of electron delocalization and the average level of double occupations.

To be explicit, we first focus on the half-filled Hubbard dimer. The average local number of double occupations according to Eqs. (5) and (9) is given by

$$
\omega_{i}=\frac{1}{2}\left(\gamma_{i i}-\frac{\gamma_{12}^{2}}{2} \frac{1+\sqrt{\gamma_{i i}\left(2-\gamma_{i i}\right)-\gamma_{12}^{2}}}{\left(1-\gamma_{i i}\right)^{2}+\gamma_{12}^{2}}\right)
$$

for $U_{i}>0$. The upper and lower bounds on the ground state $\gamma_{12}$ are obtained from the charge distribution $\gamma_{i i}$ as

$$
\gamma_{12}^{0}=\sqrt{\gamma_{i i}\left(2-\gamma_{i i}\right)}
$$

and

$$
\gamma_{12}^{\infty}= \begin{cases}\sqrt{2 \gamma_{i i}\left(1-\gamma_{i i}\right)} & \text { for } \quad \gamma_{i i} \leqslant 1 \\ \sqrt{2\left(\gamma_{i i}-1\right)\left(2-\gamma_{i i}\right)} & \text { for } \quad \gamma_{i i} \geqslant 1,\end{cases}
$$


where $0 \leqslant \gamma_{i i} \leqslant 2$. Introducing the degree of electron delocalization

$$
g_{i}^{2}=\frac{\gamma_{12}^{2}-\gamma_{12}^{\infty 2}}{\gamma_{12}^{0}-\gamma_{12}^{\infty 2}},
$$

and the level of double occupations

$$
d_{i}=\frac{\omega_{i}-\omega_{i}^{\infty}}{\omega_{i}^{0}-\omega_{i}^{\infty}},
$$

it is easy to see that Eq. (19) is equivalent to

$$
d_{i}=\frac{\left(1-\sqrt{1-g_{i}^{2}}\right)^{2}}{1-\delta_{i} \sqrt{1-g_{i}^{2}}},
$$

where $\delta_{i}=\min \left\{\gamma_{i i}, 2-\gamma_{i i}\right\}$ is the local number of carriers (electrons or holes). Notice that Eq. (24) relates $d_{i}$ and $g_{i}$, for all $\delta_{i}$, without involving any system-specific parameters. It is therefore reasonable to expect that this relation can be transferred with good accuracy to arbitrary systems, once a suitable general form for $g_{i}$ is obtained.

In order to quantify the degree of charge fluctuations from a local perspective, we consider

$$
\Gamma_{i}^{2}=\frac{2}{\gamma_{i i}\left(2-\gamma_{i i}\right)} \sum_{j \neq i, \sigma}\left|\gamma_{i j \sigma}\right|^{2} .
$$

A similar expression has been proposed in Refs. [36,37] in the context of the interaction-energy functional of the single-impurity Anderson model. $\Gamma_{i}^{2}$ is proportional to the square of the norm of the column vector $\gamma_{i j \sigma}$, for fixed $i$, excluding the diagonal terms $\gamma_{i i \sigma}$. It represents the degree of electron delocalization around the lattice site $i$ for the given $\gamma_{i j}$. Although specific to the site $i, \Gamma_{i}^{2}$ should be regarded as a semilocal property, since it carries mostly information on the immediate environment of each atom. Numerical calculations show that the ground-state value of $\Gamma_{i}$ is dominated by the contributions of the sites $j$ for which $t_{i j} \neq 0$. By definition, $\Gamma_{i}^{2}$ is invariant under unitary transformations among the orbitals $j \neq i$. Moreover, one can show that $0 \leqslant \Gamma_{i}^{2} \leqslant 1[36,37]$. Thus, $\Gamma_{i}$ reaches its maximum $\Gamma_{i}^{0^{2}}=1$ when the system is noninteracting and its minimum when there is no electron delocalization at all (i.e., $\gamma_{i j}=0$ for all $i \neq j$ ). It should be however noted, that the electrons are not fully localized in the ground state away from half-band filling, even in the strongly interacting limit $(U \rightarrow \infty)$. In general we have $\Gamma_{i} \geqslant \Gamma_{i}^{\infty}$, where $\Gamma_{i}^{\infty}$ is obtained by replacing $\gamma_{i j}^{\infty}$ in Eq. (25). This corresponds to the minimum average number of double occupations for the given charge distribution $\gamma_{i i}$.

Scaling $\Gamma_{i}^{2}$ within the domain of $v$ representability of $\gamma_{i j}$ we obtain

$$
g_{i}^{2}=\frac{\Gamma_{i}^{2}-\Gamma_{i}^{\infty 2}}{\Gamma_{i}^{0^{2}}-\Gamma_{i}^{\infty 2}} .
$$

The present generalized form of $g_{i}^{2}$ applies to all sites $i$, irrespectively of their specific local environment. It reduces to Eq. (22) in the case of a dimer. Using the dimer expression (24) for $d_{i}$ with the generalized form of $g_{i}^{2}$ given by Eq. (26), we obtain a simple universal semilocal scaling approximation
(SLSA) to the interaction-energy functional of Hubbard-like models. The local contributions entering Eq. (9) are given by

$$
\omega_{i}=\omega_{i}^{\infty}+d_{i}\left(\omega_{i}^{0}-\omega_{i}^{\infty}\right),
$$

where $\omega_{i}^{0}$ and $\omega_{i}^{\infty}$ are simple functions of $\gamma_{i i}$. In most cases, $\omega_{i}^{0}=\gamma_{i i, \uparrow} \gamma_{i i, \downarrow}=\gamma_{i i}^{2} / 4$ and $\omega_{i}^{\infty}=\max \left\{\gamma_{i i}-1,0\right\}$. In the following sections Eqs. (24)-(27) will be applied to both homogeneous and inhomogeneous models.

The accuracy of the present semilocal approximation to $W$ relies to a large extent on the transferability of the relation (24), which was originally derived from the exact dimer functional. In Fig. 2 we show exact results for $d_{i}$ as a function of $g_{i}^{2}$. These were obtained by performing Lanczos diagonalizations

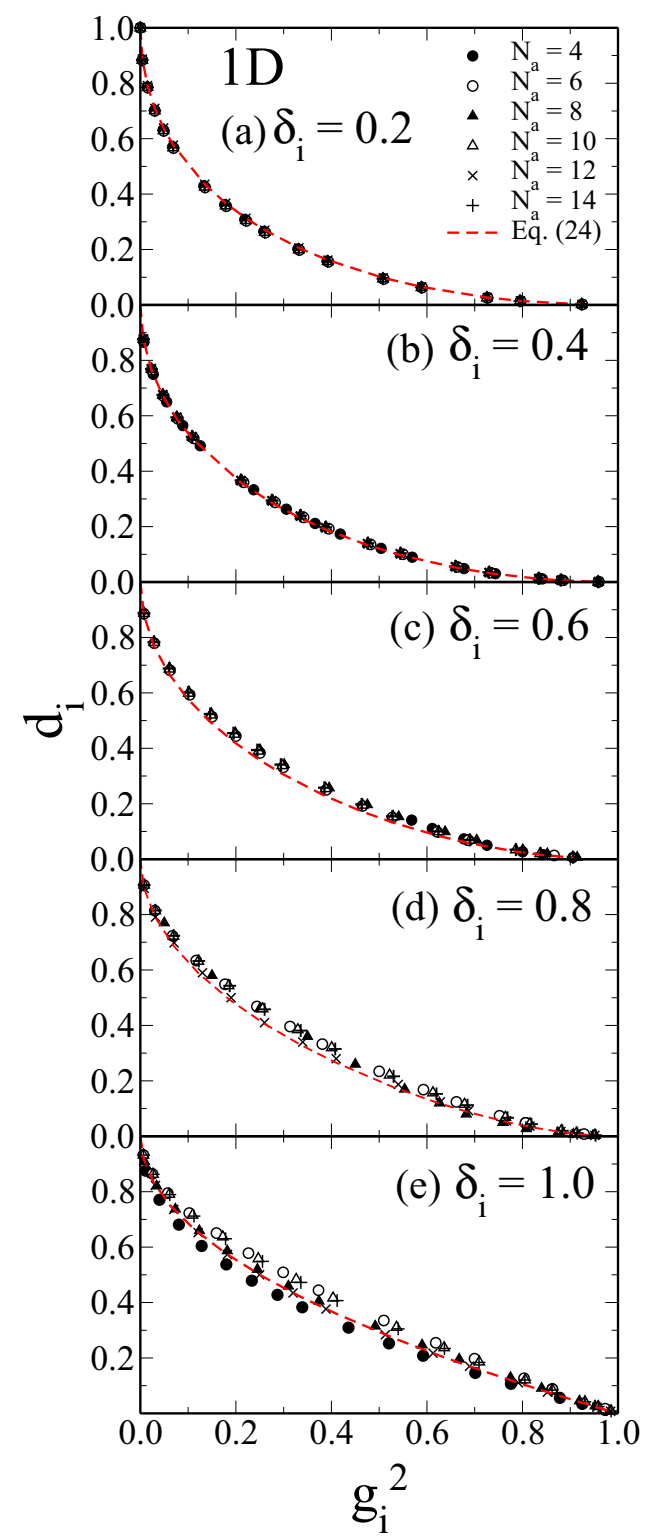

FIG. 2. Average level of double occupations $d_{i}$ as a function of the degree of electron delocalization $g_{i}^{2}$ for bipartite rings having $N_{a}=$ 4-14 atoms, band-filling $n=1$, and different local carrier densities $\delta_{i}$ [see Eqs. (23) and (26)]. The symbols are exact results obtained from Lanczos diagonalizations and the red dashed curves correspond to the scaled semilocal functional given by Eqs. (24)-(26). 


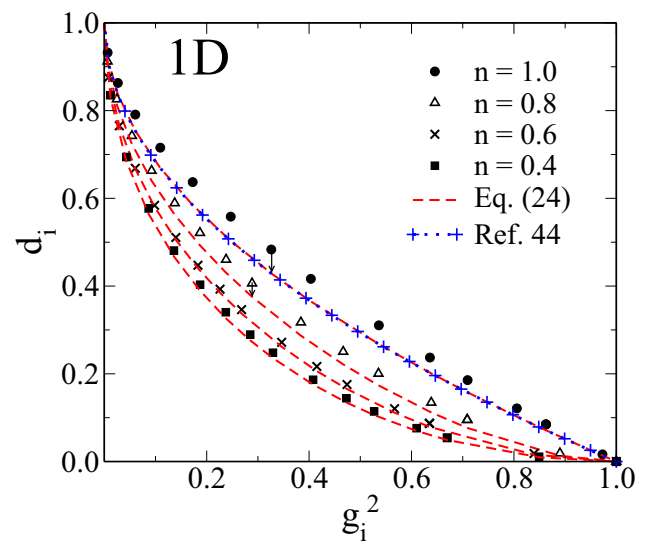

FIG. 3. Average level of double occupations $d_{i}$ as a function of the degree of electron delocalization $g_{i}^{2}$ in homogeneous Hubbard rings $\left(\varepsilon_{i}=0\right)$ having $N_{a}=10$ sites and different band fillings $n$. The symbols show exact Lanczos results, the dashed red curves the present semilocal scaling approximation [Eqs. (24)-(26)], and the blue plus signs the Ansatz proposed in Ref. [44].

[55] in a variety of one-dimensional half-filled Hubbard rings $(n=1)$ having different numbers of atoms $N_{a}$ and different local carrier densities $\delta_{i}$. One observes that the dependences of $d_{i}$ on $g_{i}^{2}$ are all remarkably similar and that the scaling approximation based on the dimer exact solution (dashed red curves) is quite accurate in general. The largest deviations are found close to the homogeneous case $\left(\delta_{i}=1\right)$. This demonstrates the pertinence of the scaling approach and lets us expect a good accuracy in the applications.

A further interesting feature of the present approach is that it takes explicitly into account the dependence of $\omega_{i}$ on the charge density distribution $\gamma_{i i}$, thereby respecting the electron-hole symmetry. This goes beyond previous scaling approximations, in which the dependence on $g_{i}$ was assumed to be the same for all carrier densities $[44,46]$. In order to quantify the importance of this contribution, Fig. 3 shows $d_{i}$ as a function of $g_{i}^{2}$ for an homogeneous Hubbard ring ( $\varepsilon_{i}=0$ ) having $N_{a}=10$ sites and different band fillings $n$. The symbols are exact numerical results obtained from Lanczos diagonalization, while the dashed red curves correspond to Eq. (24). One observes that the dependence on carrier density is significant and that the present functional is able to reproduce it quite correctly. The accuracy is thereby improved with respect to earlier approximations (blue plus signs) [44,46], to which the present approach reduces for $\gamma_{i i}=1$.

\section{HOMOGENEOUS SYSTEMS}

As a first application we consider the one-dimensional (1D) chain and the two-dimensional (2D) square lattice with nearest neighbor hoppings and a homogeneous charge distribution at half-band filling $\left(\varepsilon_{i}=0\right.$ for all $\left.i\right)$. In particular we would like to compare the present semilocal functional with highly-accurate numerical methods, such as density-matrix renormalization group (DMRG) in one dimension [57] and quantum Monte Carlo (QMC) in two dimensions [52]. In addition, it is interesting to analyze the differences between our approach and other approximate methods, such as slave-boson meanfield (SBMF) [51] which also aim to describe the interplay between electron localization and charge fluctuations in a simple way.

For a homogeneous lattice, having $\mathrm{NN}$ hoppings $t$, coordination number $z$, and local repulsions $U$, the ground-state energy of the Hubbard model can be written as

$$
\frac{E_{\mathrm{gs}}}{N_{a}}=-z q t \gamma_{12}^{0}+U \omega,
$$

where $\gamma_{12}^{0}$ is the NN density-matrix element in the uncorrelated limit $(U=0), q$ the renormalization factor yielding the correlated hopping rate $\gamma_{12}=q \gamma_{12}^{0}$, and $\omega=\left\langle n_{i \uparrow} n_{i \downarrow}\right\rangle$ the average number of double occupations at any site $i$. Alternatively one could attach the factor $q$, which describes the correlation-induced reduction of the kinetic energy, to an effective hopping integral $t^{\prime}=q t$, as it usually done in the SBMF approach [51]. In order to determine $q$ within LDFT it is convenient to approximate $g_{i}^{2} \simeq\left(\gamma_{12} / \gamma_{12}^{0}\right)^{2}$, which can be shown to be quite accurate for the ground state [48]. In this way the SLSA functional yields

$$
q=\frac{1}{\sqrt{1+(2 U / D)^{2}}},
$$

where $D=8 z t \gamma_{12}^{0}>0$ is proportional to the kinetic energy of the uncorrelated system, and

$$
\omega=\frac{1}{4}\left(1-\sqrt{1-\frac{1}{1+(2 U / D)^{2}}}\right) .
$$

On the other hand, in the spin-restricted slave-boson (SB) saddle-point approximation [51] one obtains

$$
q_{\mathrm{SB}}=1-\left(\frac{U}{D}\right)^{2}
$$

and

$$
\omega_{\mathrm{SB}}=\frac{1}{4}(1-U / D) .
$$

Significant differences between the two approaches are found even in the weakly correlated limit. Since $q=1-2(U / D)^{2}+$ $\mathcal{O}(U / D)^{4}$ and $\omega=(1-2|U / D|) / 4+\mathcal{O}(|U / D|)^{3}$ for small $U / t>0$ [see Eqs. (29) and (30)] the SLSA yields a smaller renormalization of the hopping rate $q$ and a larger average number of local double occupations $\omega$ than SBMF. Moreover, for large $U / t$, the SB saddle-point approximation predicts that both $q_{S B}$ and $\omega_{S B}$ vanish when $U$ reaches the critical value $U_{c}=D$ [51]. This implies full electron localization and has been interpreted as a Mott transition $\left(t^{\prime}=q t=0\right.$ and $\left.\omega=0\right)$. In contrast, in LDFT $q$ and the kinetic energy are never zero for finite values of $U / t$, in agreement with the exact solution. This will be discussed in more detail in the following section.

In order to compare the different methods and to contrast them with highly accurate numerical calculations, we show in Fig. 4 the ratio $E_{K} / E_{K}^{0}$ between the correlated and uncorrelated kinetic energies of homogeneous $1 \mathrm{D}$ and $2 \mathrm{D}$ lattices as a function of the Coulomb repulsion strength $U / t$. The results show that the SLSA yields sound results for all $U / t$, while spin-restricted SBMF fails for large $U / t$. However, the accuracy of SBMF is significantly improved if brokensymmetry spin-polarized solutions are allowed, particularly since the blocking of $q$ at large $U / t$ disappears [59]. In 


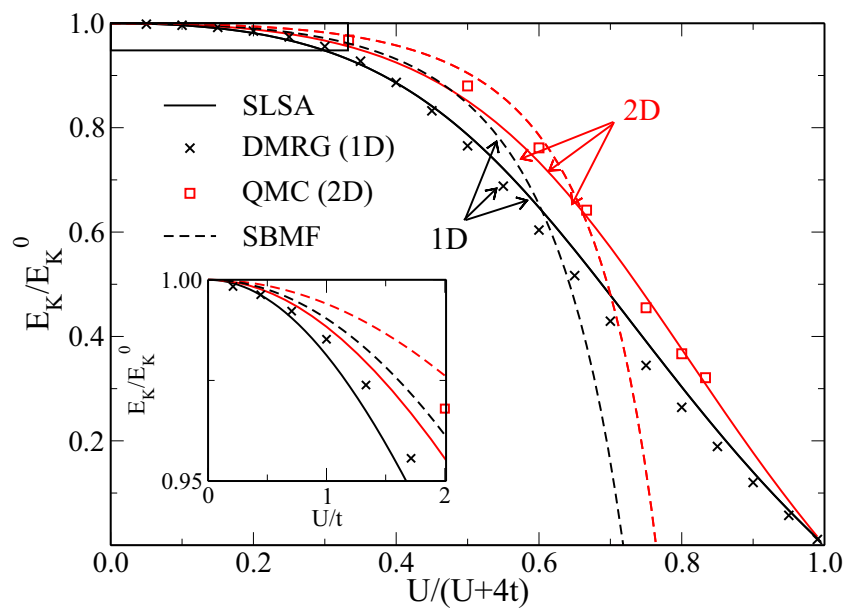

FIG. 4. Renormalization of the kinetic energy $E_{K} / E_{K}^{0}$ in the ground state of one-dimensional (1D, black curves) and twodimensional (2D square, red curves) homogeneous Hubbard models. Lattice density functional theory in the semilocal scaling approximation (SLSA, full curves) is compared with slave-boson meanfield (SBMF, dashed curves), density matrix renormalization group (DMRG, crosses [57]) and quantum Monte Carlo (QMC, squares [52]). The inset highlights the weakly correlated limit.

the weakly correlated limit one observes that LDFT-SLSA underestimates the kinetic energy renormalization, while SBMF overestimates it (see inset). This can be partly explained in terms of the lowest order expansion of $q$ as a function of $U / t$. Moreover, higher-order contributions are also important, since the SLSA functional depends explicitly on the renormalization of the hopping rate, while in SBMF the average number of double occupations depends only on the ratio $U / D$. Finally, it should be recalled that our semilocal interaction-energy functional $W$ coincides with the one introduced in Ref. [44] for the homogeneous models considered in this section. This is no longer the case for the inhomogeneous situations discussed in the following.

\section{INHOMOGENEOUS COULOMB REPULSIONS}

In Sec. II A a local formulation of the interaction-energy functional $W[\gamma]$ of the Hubbard model has been introduced, which can be applied to arbitrary on-site interactions [see Eqs. (6)-(9)]. An explicit semilocal scaling approximation (SLSA) to $W$, or equivalently to the average number of double occupations $\omega_{i}[\gamma]$ at site $i$, has been proposed in Sec. III. In order to quantify the performance of the functional in the case of inhomogeneous interactions, we consider a halffilled infinite $1 \mathrm{D}$ chain having alternating Coulomb integrals $U_{1}=\bar{U}-\Delta U / 2$ on sublattice $\mathcal{S}_{1}$ and $U_{2}=\bar{U}+\Delta U / 2$ on sublattice $\mathcal{S}_{2}$. In Fig. 5 the ground-state properties obtained with the present semilocal Ansatz are shown for $U / t=4$ and $|\Delta U| / t \leqslant 2 \bar{U} / t=8$. This covers the range $0 \leqslant U_{1}, U_{2} \leqslant 8 t$ which includes the crossover from weak to strong correlations at the two sublattices. Particularly interesting are intermediate values of $U_{i}$, where the accuracy of scaling approximations to LDFT is known to be most delicate $[47,48]$. The results of precise numerical DMRG calculations are also given for the sake of comparison [57].

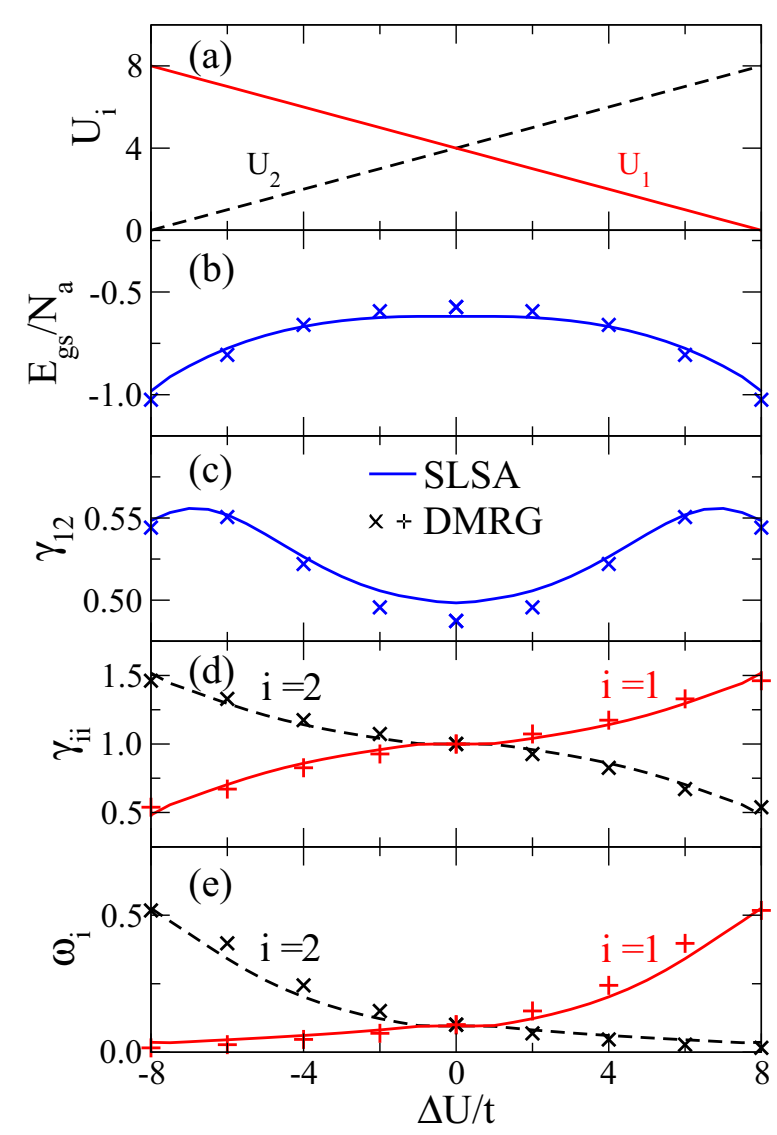

FIG. 5. Ground-state properties of the 1D Hubbard model with alternating Coulomb repulsions $U_{1}=\bar{U}-\Delta U / 2$ and $U_{2}=\bar{U}+$ $\Delta U / 2$ as a function of $\Delta U=U_{2}-U_{1}$ for $\bar{U} / t=4$ and band-filling $n=1$ [see subfigure (a)]. Results are given for (b) the ground state energy $E_{g s}$, (c) the NN density-matrix element $\gamma_{12}$, (d) the average occupation $\gamma_{i i}$ and (e) the average number of double occupations $\omega_{i}$ at the two sublattices. The curves refer to LDFT with the present semilocal scaling approximation (SLSA), while the symbols correspond to DMRG calculations [57].

One observes that $E_{g s}$ decreases and that $\gamma_{12}$ increases quadratically as $|\Delta U|$ increases. This can be qualitatively understood as the result of an enhancement of the effective exchange coupling between the localized atomic spins in the strongly correlated limit. Indeed, for $U_{i} / t \gg 1$, the half-filled Hubbard model on a bipartite lattice with interactions $U_{1}$ and $U_{2}$ can be mapped to an effective spin-1/2 Heisenberg model, which includes $\mathrm{NN}$ exchange interactions $J=2 t^{2}\left(1 / U_{1}+\right.$ $1 / U_{2}$ ) [60]. For not too large $\Delta U$ one then has $J \simeq$ $\left(4 t^{2} / \bar{U}\right)\left[1+(\Delta U / 2 \bar{U})^{2}+O(\Delta U / \bar{U})^{4}\right]$. Thus, increasing the inhomogeneity enhances the exchange coupling $J$ and lowers the ground-state energy. A similar argument explains the enhancement of the density matrix element $\gamma_{12}$ with increasing $\Delta U$. However, the strong coupling approximation breaks down when one of the $U_{i}$ is comparable or smaller than $t$. In Fig. 5 this corresponds to $|\Delta U| / t \gtrsim 6$. Here one observes a maximum in $\gamma_{12}$, which reflects a change of behavior leading eventually to electron localization and vanishing $\gamma_{12}$ in the limit of $\Delta U \gg$ $\bar{U}$. Notice that the proposed semilocal functional reproduces the nearly exact numerical DMRG results remarkably well. The same holds for the charge distribution $\gamma_{i i}$ and the average 
number of double occupations $\omega_{i}$ at the two sublattices. Here we observe a charge transfer towards the sublattice having the smallest $U_{i}$, as well as small reduction (important enhancement) of $\omega_{i}$ at the sites having a higher (lower) $U_{i}$. A similar very good accuracy of the SLSA has been also found in finite $1 \mathrm{D}$ and $2 \mathrm{D}$ clusters with periodic boundary conditions by comparison with exact Lanczos diagonalizations.

\section{CROSSOVER FROM BAND TO MOTT INSULATORS}

In this section we consider the bipartite ionic Hubbard model given by

$$
\hat{H}=\sum_{i, \sigma} \varepsilon_{i} \hat{n}_{i \sigma}+t \sum_{\langle i, j\rangle \sigma} \hat{c}_{i \sigma}^{\dagger} \hat{c}_{j \sigma}+U \sum_{i} \hat{n}_{i \downarrow} \hat{n}_{i \uparrow},
$$

where $\varepsilon_{i}=\varepsilon / 2>0\left(\varepsilon_{i}=-\varepsilon / 2\right)$ for $i$ belonging to the sublattice $\mathcal{S}_{1}\left(\mathcal{S}_{2}\right)$. Considerable efforts have already been devoted to analyze the physics of this Hamiltonian, particularly at half-band filling, since for finite bipartite potential $\varepsilon$ it undergoes a transition from a band insulator to a Mott insulator as $U / t$ increases. For some particular systems this transition involves crossing an intermediate metallic phase. Examples of this situation are the 2D square lattice, as shown by using QMC methods [61], or the 2D Bethe lattice, which has been investigated using dynamical mean-field theory [62]. One-dimensional bipartite systems with NN hopping do not present a metallic phase in generalized ionic Hubbard models of the form $A B_{n}[63,64]$. This phase is expected only when next NN hoppings are included [65].

In Figs. 6 and 7 results are given for the 1D chain and the $2 \mathrm{D}$ square lattice as a function of the Coulomb repulsion strength $U / t$. Representative values of the bipartite potential $\varepsilon$ are considered at half-band filling $n=1$. LDFT with the semilocal scaling functional is compared with our DMRG calculations on the 1D chain [57], and with variational Monte Carlo (VMC) and QMC results on the 2D lattice reported in Refs. $[66,67]$. In the $1 D$ case the LDFT-SLSA results are in very good agreement with the DMRG ones in the whole range of parameters $U / t$ and $\varepsilon$. In the $2 \mathrm{D}$ case, the quantitative comparisons with VMC and QMC concern only $E_{g s}$ for the homogeneous case $(\varepsilon=0)$. Although this is usually the most difficult case, the accuracy of the SLSA is also very satisfactory. This is remarkable taking into account that performing such accurate many-body simulations is quite involved.

The dependence of the ground-state properties on $U / t$ is qualitatively similar to the one observed in $1 \mathrm{D}$ rings having $N_{a}=14$ sites [49]. An advantage of the present local formulation is that it allows us to discern the average number of double occupations $\omega_{i}$ at different sublattices [see Figs. 6(d) and $7(\mathrm{~d})]$. This is an improvement with respect to previous global approaches. For finite $\varepsilon$ an interesting redistribution of the double occupations $\omega_{i}$ is observed as a function of $U / t$. On the sublattice $\mathcal{S}_{2}$, having the lower energy level $\varepsilon_{2}=-\varepsilon / 2<0, \omega_{2}$ decreases with increasing $U / t$ in order to lower the Coulomb energy. At the same time the electron density is redistributed. The charge transfer $\Delta n$ becomes smaller, since an homogeneous charge distribution reduces the average number of double occupations. See subfigures (b) and (d) of Figs. 6 and 7, keeping in mind that $\omega_{2}$ has been plotted along the negative axis for the sake of compactness.

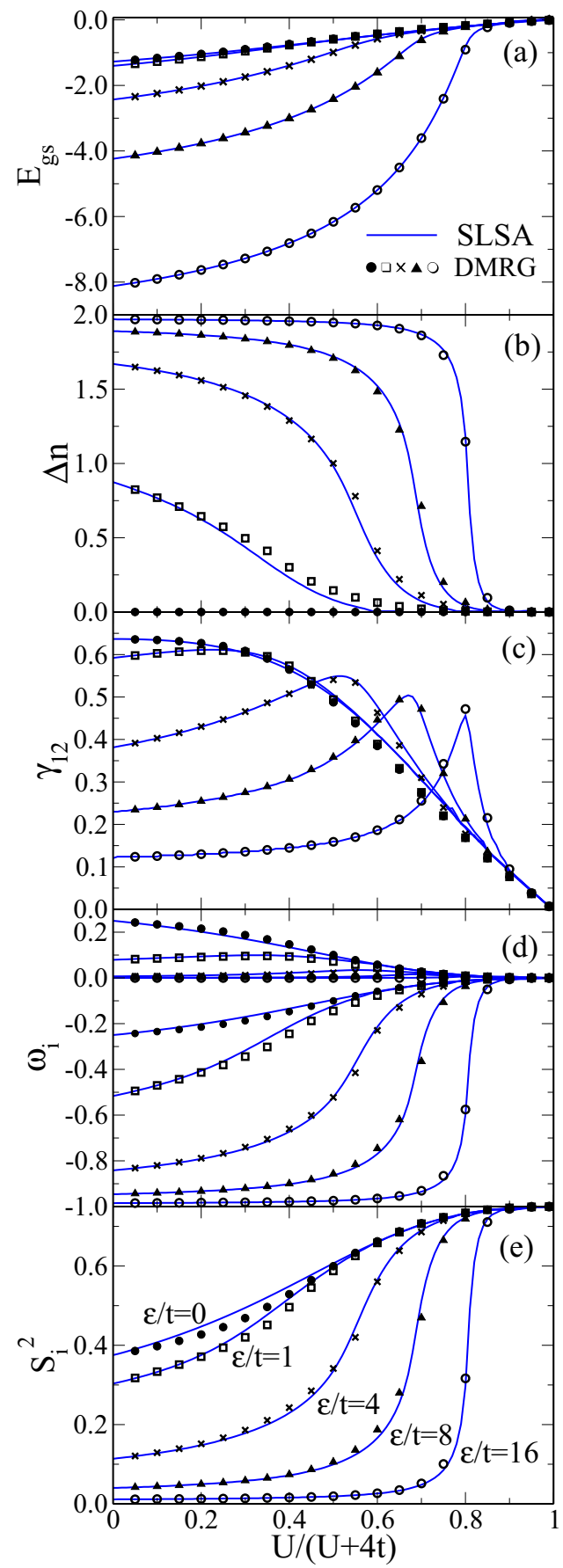

FIG. 6. Ground-state properties of 1D Hubbard chains at halfband filling $n=1$ as a function of Coulomb repulsion strength $U / t$ for representative bipartite potentials $\varepsilon$ [see Eq. (33)]. Results are given for (a) the ground states energy $E_{g s}$, (b) the charge transfer $\Delta n=\gamma_{22}-\gamma_{11}$ between the two sublattices, (c) the NN densitymatrix elements $\gamma_{12}$, (d) the average number of double occupations $\omega_{1}$ on sublattice $\mathcal{S}_{1}$ having $\varepsilon_{1}=\varepsilon / 2>0$ (positive values), $-\omega_{2}$ corresponding to sublattice $\mathcal{S}_{2}$ which has $\varepsilon_{2}=-\varepsilon / 2<0$ (negative values), and finally (e) the local spin momentum $S_{i}^{2}$. Solid curves refer to LDFT in the semilocal scaling approximation (SLSA), while the symbols to DMRG [57].

Notice that the kinetic energy is also lowered by increasing $\gamma_{12}\left[t<0\right.$, see (c)]. On the less favorable sublattice $\mathcal{S}_{1}, \omega_{1}$ shows a nonmonotonous dependence on $U / t$. Starting from 


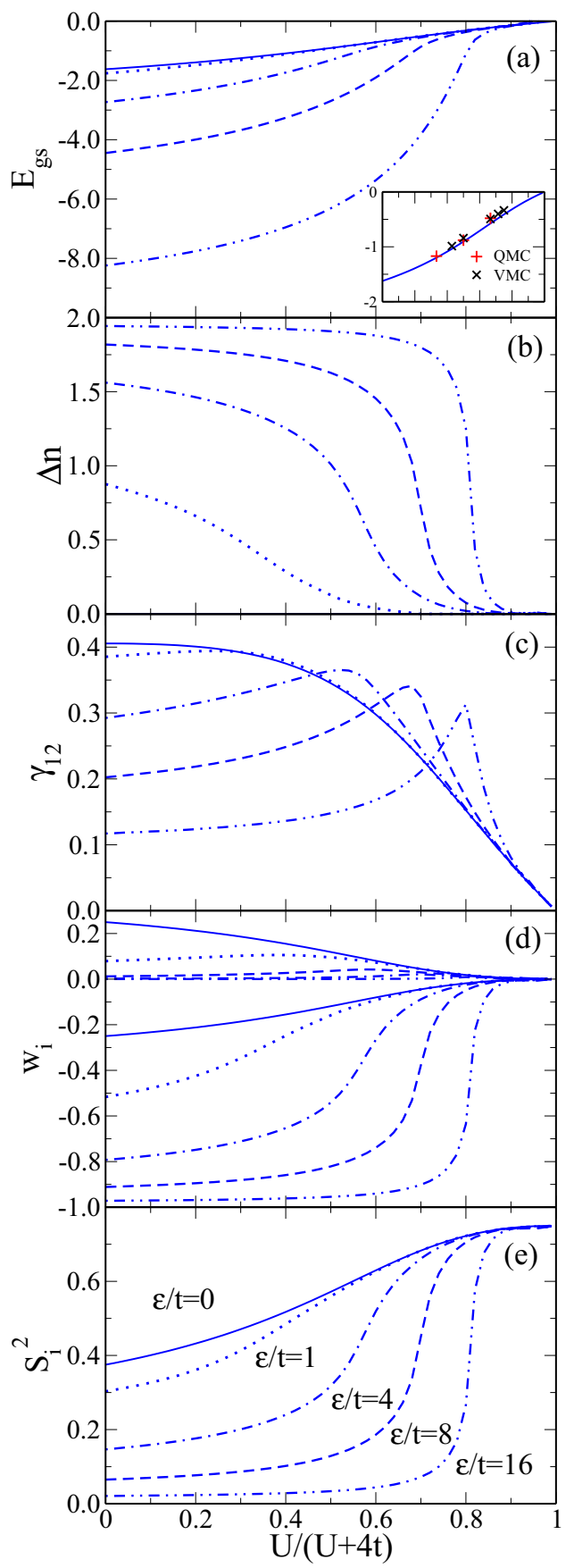

FIG. 7. Lattice density-functional theory results for the groundstate properties of the ionic Hubbard model on the 2D square lattice as in Fig. 6. In the inset of (a) LDFT-SLSA (full curve) is compared to quantum Monte Carlo simulations (red plus signs, Ref. [67]) and variational Monte Carlo simulations (blue crosses, Ref. [66]).

the uncorrelated limit, $\omega_{1}$ first increases with increasing $U / t$ as a result of the increase in local density $\gamma_{11}$ (i.e., the decrease of $\Delta n$ ). However, when $U / t$ becomes more important, charge fluctuations and double occupations start to be suppressed also on $\mathcal{S}_{1}$, already before a nearly uniform charge distribution is established [see (b)]. This leads to a decrease of $\omega_{1}$, which thus shows a maximum at a finite $U / t$.

The local magnetic moments $S_{i}^{2}=\frac{3}{4}\left\langle\left(\hat{n}_{i \uparrow}-\hat{n}_{i \downarrow}\right)^{2}\right\rangle=$ $\frac{3}{4}\left(\gamma_{i i}-2\left\langle\hat{n}_{i \uparrow} \hat{n}_{i \downarrow}\right\rangle\right)$ at site $i$, shown in Figs. 6(e) and 7(e),

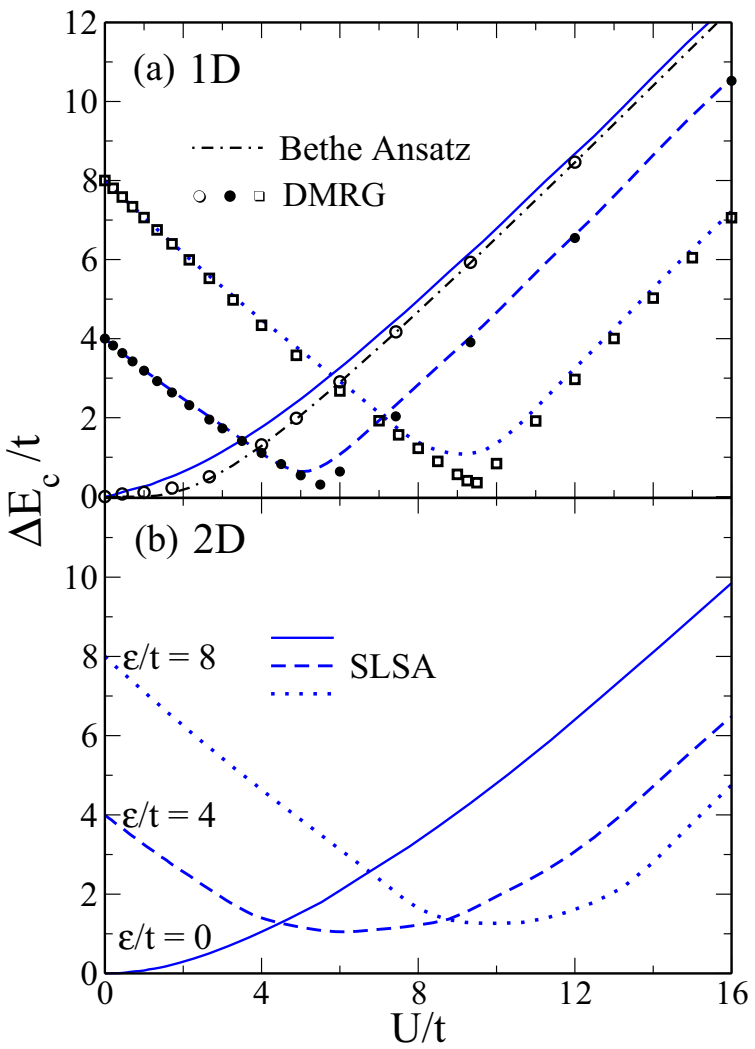

FIG. 8. Charge gap $\Delta E_{\mathrm{c}}$ in the half-filled ionic Hubbard model for (a) the $1 \mathrm{D}$ chain and (b) the 2D square lattice as a function of the Coulomb repulsion strength $U / t$. The curves are obtained by using LDFT with the semilocal scaling functional (SLSA), for representative values of the bipartite potential $\varepsilon / t$ as indicated. In the $1 \mathrm{D}$ case comparison is made with DMRG calculations (symbols [57]) and the Bethe ansatz solution ( $\varepsilon=0$, dash-dotted curve).

provide a complementary perspective to the electronic correlations as a function of $\varepsilon / t$ and $U / t$. In the case of half-band filling, $S_{i}$ is the same on the two sublattices due to electronhole symmetry, even in the presence of charge transfer. The proposed functional fulfills this symmetry condition strictly. Indeed, using Eqs. (24) and (27) for $n=1$, one obtains $\omega_{2}-\omega_{1}=\omega_{2}^{\infty}-\omega_{1}^{\infty}$ and then $S_{2}^{2}=\frac{3}{4}\left(\gamma_{22}-2 \omega_{2}\right)=$ $\frac{3}{4}\left(\gamma_{11}-2 \omega_{1}\right)=S_{1}^{2}$. In the uncorrelated limit we have $\hat{n}_{i \uparrow} \hat{n}_{i \downarrow}=$ $\gamma_{i i}^{2} / 4$ and therefore $S_{i}^{2}$ depends only on the density distribution $\gamma_{i i}$. In particular at half-band filling, $S_{i}^{2}$ decreases with increasing $\varepsilon / t$, from $S_{i}^{2}=3 / 8$ for $\varepsilon / t=0$, to $S_{i}^{2}=0$ for $\varepsilon / t=\infty$. In the latter case all electrons are paired on one sublattice. As $U / t$ increases, the charge imbalance is reduced and $S_{i}^{2}$ increases for $n=1$ [see Figs. 6(e) and 7(e)]. Finally, for $U \gg t$ and $\varepsilon$, the largest possible $S_{i}^{2}=3 / 4$ is obtained, since all sites are then singly occupied regardless of $\varepsilon / t$.

In Fig. 8 the charge gap

$$
\Delta E_{\mathrm{c}}=E\left(N_{\mathrm{e}}+1\right)+E\left(N_{\mathrm{e}}-1\right)-2 E\left(N_{\mathrm{e}}\right)
$$

is shown as a function of $U / t$ in half-filled 1D and 2D lattices for representative energy-level shifts $\varepsilon / t$. It is well known that no metal-insulator transition occurs at a finite value of $U / t$ in one- and two-dimensional homogeneous bipartite lattices $(\varepsilon=$ $0)$. In fact, $\Delta E_{\mathrm{c}}>0$ already for arbitrary small $U / t$. Only in 
the absence of interaction and inhomogeneity $(U=\varepsilon=0)$ the system is metallic ( $\left.\Delta E_{\mathrm{c}}=0\right)$. On the one side, for $U=0$, any finite $\varepsilon / t$ opens a gap in the one-particle spectrum of bipartite lattices, which behave as a conventional band insulator $(n=$ $1)$. On the other side, in the strongly correlated limit $(U \gg t$ and $\varepsilon$ ) $\Delta E_{\mathrm{c}}$ increases linearly with $U / t$ for all $\varepsilon$. In this case all sites are singly occupied (large $U / t$ and $n=1$ ). Therefore, adding an electron inevitably implies an additional double occupation, while removing one does not. The gap is thus a direct consequence of the local interactions, and the system is said to be a Mott insulator. Finally, when $U$ and $\varepsilon$ are both finite, a transition or crossover from band to Mott regimes and a nonmonotonous dependence of $\Delta E_{\mathrm{c}}$ are observed as a function of $U / t$.

In the absence of interactions we have $\Delta E_{\mathrm{c}}=\varepsilon$ for $n=1$, both in $1 \mathrm{D}$ and $2 \mathrm{D}$ bipartite lattices. Increasing $U / t$ results in a reduction of $\Delta E_{\mathrm{c}}$, which initially depends linearly on $U$, approximately as $\Delta E_{\mathrm{c}} \sim \varepsilon-U$ for not too small $\varepsilon$ (see Fig. 8). This can be understood by noting that the Coulomb repulsion on sublattice $\mathcal{S}_{2}$ compensates the single-particle energy difference $\varepsilon$ between the two sublattices, rendering doubly occupied $\mathcal{S}_{2}$ sites and singly-occupied $\mathcal{S}_{1}$ sites closer in energy. In this way, as $U$ increases, the delocalization of the $\gamma_{11}$ electrons in $\mathcal{S}_{1}$, as well as the $\gamma_{22}-1$ extra electrons on $\mathcal{S}_{2}$, is favored $\left(\gamma_{11}<\gamma_{22}\right.$ for $\left.\varepsilon>0\right)$. Consequently, the energy difference between adding and removing an electron decreases until $U$ is of the order of $\varepsilon$. A minimum in $\Delta E_{\mathrm{c}}$ is reached for $U / t$ somewhat larger but of the order of $\varepsilon / t$, since for $U>\varepsilon$ the tendency inverts itself. Indeed, for $U$ beyond the minimum, $\Delta E_{\mathrm{c}}$ grows monotonously as the strongly correlated regime is approached. Notice that the transition region, where $\Delta E_{\mathrm{c}}$ remains close to its minimum value, is significantly broader in two dimensions than in one dimension. This can be ascribed to the stronger kinetic energy and bandwidth in the former. For these intermediate values of $U / t$ the system is nearly metallic, since the charge gap is small. In fact, QMC simulations predicts a metallic phase in two dimensions [61], which we do not observe with the present approximated functional. Nevertheless, the minimum of the charge gap is a useful indication of a transition or crossover from band to Mott insulator. Finally, when the Coulomb repulsion energy becomes larger than the kinetic and charge-transfer energies $(U \gg t$ and $\varepsilon$ ) the electrons become localized on both sublattices. Thus, $\Delta E_{\mathrm{c}}$ increases linearly with $U / t$ as in the homogeneous case.

Comparing the present LDFT-SLSA results in one dimension with the Bethe-Ansatz solution $(\varepsilon=0)$ and DMRG calculations one observes a very good quantitative agreement, except in the admittedly most interesting metallic or nearly metallic regime, where $\Delta E_{\mathrm{c}}$ is significantly overestimated. Even in the homogeneous case, where the gap correctly vanishes at $U=0$, the scaling functional predicts $\Delta E_{\mathrm{c}} \propto(U / t)^{2}$, in contrast to the exact exponential dependence obtained with the Bethe ansatz [29,30] (see Fig. 8). For $\varepsilon / t \neq 0$, the critical value $U_{c}$ of the Coulomb repulsion, for which $\Delta E_{\mathrm{c}}$ is minimal, is somewhat underestimated with our functional. For example, for $\varepsilon / t=4$ we obtain $U_{c}^{\mathrm{LDFT}} / t \simeq 5.2$ while $U_{c}^{\mathrm{DMRG}} / t \simeq 5.5$. However, the most important difference between the SLSA and DMRG results is the overestimation of the gap near the critical point. This is consistent with the fact that no metallic phase is found between the band- and Mott-insulator phases in two dimensions, in contrast to QMC simulations. The overestimation of $\Delta E_{\mathrm{c}}$ close to the metallic phases is probably a consequence of extracting the functional dependence of $\omega_{i}[\gamma]$ from the dimer or, more generally, it may reflect difficulties in describing long-range effects. The dimer, having a discrete spectrum, shows a charge gap proportional to $(U / t)^{2}$ in the weakly correlated limit. This handicaps the present scaling approach which uses the dimer as a reference system.

In conclusion, a local formulation of the interaction-energy functional $W[\gamma]$ of the Hubbard model has been introduced, which can be applied to arbitrary on-site interactions. An explicit semilocal approximation to the interaction-energy functional $W[\gamma]$ of Hubbard-like models has been proposed by taking advantage of its scaling behavior and combining it with exact analytic results for the Hubbard dimer. From a local perspective, this approach can be regarded as an effective medium theory, since the effects of the environment on each site $i$ are mapped into a single additional orbital. The contribution of site $i$ to the interaction energy is then calculated within this two-level system, which has the correct charge density $\gamma_{i i}$ and a renormalized effective degree of electronic delocalization $g_{i}^{2}$. Despite its simplicity, the method captures the main physics of the interplay between electron delocalization, charge redistribution, and Coulomb repulsion in the ionic Hubbard model. Important changes in the groundstate properties are well reproduced for homogeneous and inhomogeneous single-particle potentials and local Coulomb interactions, including the crossover from band insulator (charge density wave) to Mott insulator (homogeneous localized charge). The calculated ground-state energy $E_{\mathrm{gs}}, \mathrm{NN}$ bond order $\gamma_{12}$, charge transfer between sublattices $\Delta n$, correlation energy, and local spin moments are in very good quantitative agreement with accurate numerical and analytical solutions. The band gap is, however, significantly overestimated in the vicinity of the intermediate metallic or nearly metallic regime. Further developments of the interaction-energy functional are therefore worthwhile.

The possibility of treating inhomogeneous local interactions opens the way to a number of interesting studies of the interplay between strong and moderate local interactions in the framework of LDFT. Using the functional proposed in this work, one could investigate single-impurity models by taking into account the effects of nonvanishing Coulomb repulsions in the conduction band. In addition, one could look into periodic Anderson-like models including localized and delocalized orbitals with different local interactions. Finally, one should examine 1D and 2D interfaces between a strongly correlated system and a free-electron or weakly correlated metal (e.g., wires or thin films deposited on surfaces). This would provide us with an alternative perspective to the proximity-induced correlation effects.

\section{ACKNOWLEDGMENTS}

Helpful discussion with T. Müller and W. Töws are gratefully acknowledged. Computer resources were provided by the IT Service Center of the University of Kassel and by the Center for Scientific Computing of the University of Frankfurt. 
[1] P. Hohenberg and W. Kohn, Phys. Rev. 136, B864 (1964).

[2] W. Kohn and L. J. Sham, Phys. Rev. 140, A1133 (1965).

[3] U. von Barth and L. Hedin, J. Phys. C 5, 1629 (1972).

[4] R. G. Parr and W. Yang, Density-Functional Theory of Atoms and Molecules (Oxford University Press, Oxford, 1989); R. M. Dreizler and E. K. U. Gross, Density Functional Theory (Springer, Berlin, 1990).

[5] D. C. Langreth and M. J. Mehl, Phys. Rev. B 28, 1809 (1983); A. D. Becke, Phys. Rev. A 38, 3098 (1988); J. P. Perdew, K. Burke, and M. Ernzerhof, Phys. Rev. Lett. 77, 3865 (1996).

[6] J. Toulouse, I. C. Gerber, G. Jansen, A. Savin, and J. G. Ángyán, Phys. Rev. Lett. 102, 096404 (2009); J. Toulouse, W. Zhu, J. G. Ángyán, and A. Savin, Phys. Rev. A 82, 032502 (2010); W. Zhu, J. Toulouse, A. Savin, and J. G. Ángyán, J. Chem. Phys. 132, 244108 (2010).

[7] B. G. Janesko, T. M. Henderson, and G. E. Scuseria, J. Chem. Phys. 130, 081105 (2009); R. M. Irelan, T. M. Henderson, and G. E. Scuseria, ibid. 135, 094105 (2011).

[8] A. D. Becke, J. Chem. Phys. 98, 5648 (1993).

[9] J. P. Perdew, M. Ernzerhof, and K. Burke, J. Chem. Phys. 105, 9982 (1996).

[10] J. Heyd, G. E. Scuseria, and M. Ernzerhof, J. Chem. Phys. 118, 8207 (2003).

[11] T. L. Gilbert, Phys. Rev. B 12, 2111 (1975).

[12] A. M. K. Müller, Phys. Lett. A 105, 446 (1984).

[13] S. Goedecker and C. J. Umrigar, Phys. Rev. Lett. 81, 866 (1998).

[14] E. J. Baerends, Phys. Rev. Lett. 87, 133004 (2001); M. A. Buijse and E. J. Baerends, Mol. Phys. 100, 401 (2002).

[15] O. Gritsenko, K. Pernal, and E. J. Baerends, J. Chem. Phys. 122, 204102 (2005); D. R. Rohr, K. Pernal, O. V. Gritsenko, and E. J. Baerends, ibid. 129, 164105 (2008).

[16] M. Piris, Int. J. Quantum Chem. 106, 1093 (2006); M. Piris, X. Lopez, F. Ruipérez, J. M. Matxain, and J. M. Ugalde, J. Chem. Phys. 134, 164102 (2011).

[17] M. A. L. Marques and N. N. Lathiotakis, Phys. Rev. A 77, 032509 (2008); N. N. Lathiotakis and M. A. Marques, J. Chem. Phys. 128, 184103 (2008).

[18] N. N. Lathiotakis, S. Sharma, J. K. Dewhurst, F. G. Eich, M. A. L. Marques, and E. K. U. Gross, Phys. Rev. A 79, 040501(R) (2009).

[19] D. R. Rohr, J. Toulouse, and K. Pernal, Phys. Rev. A 82, 052502 (2010).

[20] E. Dagotto, Rev. Mod. Phys. 66, 763 (1994); A. Georges, G. Kotliar, W. Krauth, and M. J. Rozenberg, ibid. 68, 13 (1996); M. Imada, A. Fujimori, and Y. Tokura, ibid. 70, 1039 (1998).

[21] K. N. Kudin, G. E. Scuseria, and R. L. Martin, Phys. Rev. Lett. 89, 266402 (2002).

[22] I. D. Prodan, G. E. Scuseria, and R. L. Martin, Phys. Rev. B 73, 045104 (2006); 76, 033101 (2007).

[23] X.-D. Wen et al., J. Chem. Phys. 137, 154707 (2012).

[24] V. Eyert, Phys. Rev. Lett. 107, 016401 (2011).

[25] F. Iori, M. Gatti, and A. Rubio, Phys. Rev. B 85, 115129 (2012).

[26] See, for instance, P. Fulde, Electron Correlations in Molecules and Solids (Springer, Berlin, 1991); H. March, Electron Correlation in Molecules and Condensed Phases (Plenum, New York, 1996); G. D. Mahan, Many-Particle Physics (Kluver, New York, 2000).

[27] P. W. Anderson, Phys. Rev. 124, 41 (1961).
[28] J. Hubbard, Proc. R. Soc. London A 276, 238 (1963); 281, 401 (1964); J. Kanamori, Prog. Theo. Phys. 30, 275 (1963); M. C. Gutzwiller, Phys. Rev. Lett. 10, 159 (1963).

[29] E. H. Lieb and F. Y. Wu, Phys. Rev. Lett. 20, 1445 (1968).

[30] H. Shiba, Phys. Rev. B 6, 930 (1972).

[31] Y. Nagaoka, Solid State Commun. 3, 409 (1965).

[32] H. Tasaki, Phys. Rev. B 40, 9192 (1989).

[33] E. H. Lieb, Phys. Rev. Lett. 62, 1201 (1989); 62, 1927 (1989).

[34] O. Gunnarsson and K. Schönhammer, Phys. Rev. Lett. 56, 1968 (1986); A. Svane and O. Gunnarsson, Phys. Rev. B 37, 9919 (1988); K. Schönhammer, O. Gunnarsson, and R. M. Noack, ibid. 52, 2504 (1995).

[35] A. Schindlmayr and R. W. Godby, Phys. Rev. B 51, 10427 (1995).

[36] W. Töws and G. M. Pastor, Phys. Rev. B 83, 235101 (2011).

[37] W. Töws and G. M. Pastor, Phys. Rev. B 86, 245123 (2012).

[38] P. E. Blöchl, C. F. J. Walther, and T. Pruschke, Phys. Rev. B 84, 205101 (2011).

[39] A. E. Carlsson, Phys. Rev. B 56, 12058 (1997); R. G. Hennig and A. E. Carlsson, ibid. 63, 115116 (2001).

[40] N. A. Lima, M. F. Silva, L. N. Oliveira, and K. Capelle, Phys. Rev. Lett. 90, 146402 (2003).

[41] C. Verdozzi, Phys. Rev. Lett. 101, 166401 (2008).

[42] J.-H. Hu, J.-J. Wang, G. Xianlong, M. Okumura, R. Igarashi, S. Yamada, and M. Machida, Phys. Rev. B 82, 014202 (2010).

[43] M. Saubanère and G. M. Pastor, Phys. Rev. B 90, 125128 (2014).

[44] R. López-Sandoval and G. M. Pastor, Phys. Rev. B 61, 1764 (2000).

[45] R. López-Sandoval and G. M. Pastor, Phys. Rev. B 66, 155118 (2002).

[46] R. López-Sandoval and G. M. Pastor, Phys. Rev. B 67, 035115 (2003).

[47] R. López-Sandoval and G. M. Pastor, Phys. Rev. B 69, 085101 (2004).

[48] M. Saubanère and G. M. Pastor, Phys. Rev. B 79, 235101 (2009).

[49] M. Saubanère and G. M. Pastor, Phys. Rev. B 84, 035111 (2011).

[50] S. R. White, Phys. Rev. Lett. 69, 2863 (1992); Phys. Rev. B 48, 10345 (1993).

[51] G. Kotliar and A. E. Ruckenstein, Phys. Rev. Lett. 57, 1362 (1986).

[52] S. R. White, D. J. Scalapino, R. L. Sugar, E. Y. Loh, J. E. Gubernatis, and R. T. Scalettar, Phys. Rev. B 40, 506 (1989).

[53] W. Töws, M. Saubanère, and G. M. Pastor, Theor. Chem. Acc. 133, 1422 (2014).

[54] J. F. Janak, Phys. Rev. B 18, 7165 (1978).

[55] C. Lanczos, J. Res. Nat. Bur. Stand. 45, 255 (1950); B. N. Parlett, The Symmetric Eigenvalue Problem (Prentice Hall, Engelwood Cliffs, 1980).

[56] It has been found that $T_{a}=\max \{|4 t|, n U\}$ is an efficient choice over a wide range of band fillings and Coulomb repulsion strengths. T. Müller, private communication.

[57] The DMRG calculations have been performed by using the algorithm proposed in Ref. [50]. At each renormalization group (RG) iteration $N$ the number of atoms $l$ in the chain is increased by $2(l=2 N+2)$. The side blocks are spanned by $m=400$ states, which is by far large enough to ensure a very good accuracy. The ground state is determined by means of a Davidson method. After $N=40$ initial RG iterations, we perform 20 additional RG steps and extrapolate the ground-state energy per atom $E$ by means of a least-square fit of the form $E_{l}=E+a / l$ 
based on the last 20 steps. We have checked that the $1 / l$ law is extremely accurate. The procedure is repeated adding $20 \mathrm{RG}$ steps at a time until the relative deviations in $E$ and $a$ are less than $\delta=10^{-11}$. See also Ref. [58].

[58] M. B. Lepetit and G. M. Pastor, Phys. Rev. B 56, 4447 (1997).

[59] L. Lilly, A. Muramatsu, and W. Hanke, Phys. Rev. Lett. 65, 1379 (1990); T. Li, P. Wölfle, and P. J. Hirschfeld, Phys. Rev. B 40, 6817 (1989).

[60] J. R. Schrieffer and P. A. Wolff, Phys. Rev. 149, 491 (1966).

[61] K. Bouadim, N. Paris, F. Hébert, G. G. Batrouni, and R. T. Scalettar, Phys. Rev. B 76, 085112 (2007).
[62] A. Garg, H. R. Krishnamurthy, and M. Randeria, Phys. Rev. Lett. 97, 046403 (2006).

[63] M. E. Torio, A. A. Aligia, G. I. Japaridze, and B. Normand, Phys. Rev. B 73, 115109 (2006).

[64] S. R. Manmana, V. Meden, R. M. Noack, and K. Schönhammer, Phys. Rev. B 70, 155115 (2004).

[65] G. I. Japaridze, R. Hayn, P. Lombardo, and E. Müller-Hartmann, Phys. Rev. B 75, 245122 (2007).

[66] H. Yokohama and H. Shiba, J. Phys. Soc. Jpn. B 56, 3582 (1987).

[67] J. E. Hirsch, Phys. Rev. B 31, 4403 (1985). 